\title{
NON-SURGICAL AND SURGICAL TREATMENT OF LUMBAR DEGENERATIVE DISC DISEASE IN THE COURSE OF PERIPHERAL NEUROPATHY
}

\author{
Gustaw Wójcik $^{1,2}$, Jolanta Piskorz ${ }^{3,4}$, Joanna Iłżecka ${ }^{4}$, Dorota Nalepa ${ }^{5}$
}

\author{
${ }^{1}$ Department of Imaging Diagnosis, Zofia Tarnowska from the Zamoyskis Provincial Hospital in Tarnobrzeg \\ ${ }^{2}$ Department of Balneotherapy, Chair of Rehabilitation, Physiotherapy and Balneotherapy, \\ Medical University of Lublin \\ ${ }^{3}$ Department of Intensive Care, Zofia Tarnowska from the Zamoyskis Provincial Hospital in Tarnobrzeg \\ ${ }^{4}$ Independent Neurological Rehabilitation Unit, Chair of Rehabilitation, Physiotherapy and Balneotherapy, \\ Medical University of Lublin \\ ${ }^{5}$ Department of Neurology, Cardinal Stefan Wyszyński Regional Specialist Hospital in Lublin
}

Wójcik G., Piskorz J., Iłżecka J., Nalepa D. (2015), Non-surgical and surgical treatment of lumbar degenerative disc disease in the course of peripheral neuropathy. Health Problems of Civilization, 3 (9), p. 24-30.

\begin{abstract}
Summary: The aim of this article is to review current reports pertaining the effects of treating patients with diagnosed degenerative disc disease in the lumbosacral section of the spine.

Materials and methods: the method of descriptive analysis was used. The research was conducted on the basis of materials from MEDLINE and EMBASE databases from the last ten years. The cases of patients with sciatic neuritis caused by degenerative disc disease undergoing conservative treatment, and similar group of patients treated surgically, were analysed.

Conclusions: Conservative treatment lowers the intensity of pain, but does not improve the functional abilities of the spine. Surgical treatment gives good and quick therapeutic results, but its long-term effects do not seem better than in the case of conservative treatment. Moreover, surgical treatment may result in serious complications.

In rare cases the condition results in a severe impairment of motor functions in legs and sphincter paralysis. Such patients should undergo immediate surgical treatment.
\end{abstract}

Keywords: degenerative disc disease, sciatic neuritis, surgical treatment, conservative treatment

\section{Introduction}

Degenerative disc disease, especially in the lumbar section, is the most common condition of the spine in persons with involvement or impairment of the neural system. In about $90 \%$ of the cases, sciatic neuritis is caused by disc herniation with a compression of the nerve root (Koes et al. 2007).

In the beginning of the last century (1909) Oppenheim and Kruze published a report on two cases of spine surgery caused by lumbar degenerative disc disease (Stienen et al. 2013). It was then proven that there is a correlation between the tearing of the intervertebral disc and the irritation of nerve structures inside the spinal canal (Rüttimann 1990). In their reports, Mixter and Barr underlined the inseparable link between the herniation of the nucleus pulposus and sciatic neuritis, explaining the etiopathogenesis of neurological symptoms (Mixter, Barr 1934).

The tearing of the intervertebral disc, leading to its bulging, is usually connected to a genetic deficiency that results in a low quality of sulphur bondings in collagen fibres forming the annulus fibrosus of the intervertebral disc (Zhang 2008).

The bulge on the intervertebral disc compresses the nerve tissue inside the spinal canal and causes its mechanic irritation, which leads to impairment of the blood flow and, consequently, to swelling. Degenerative disc disease results in repeated compression of the nerve roots. The roots are not surrounded by a protective layer of connective tissue which would increase its resistance to mechanic factors. Even minor compression

Address for correspondence: Gustaw Wójcik, Department of Imaging Diagnosis, Zofia Tarnowska from the Zamoyskis Provincial Hospital in Tarnobrzeg, Szpitalna 1, 39-400 Tarnobrzeg, e-mail: gustaww@tlen.pl, phone: + 48158123001

Tables: 0 Figures: 0 References: 36 Full-text PDF www.hpc.edu.pl Copyright @ Pope John Paul II State School of Higher Education in Biała Podlaska, Sidorska 95/97, 21-500 Biała Podlaska Indexation: Index Copernicus, AGRO, ProQuest, Polish Medical Bibliography, Polish Ministry of Science and Higher Education. This is an open-access article distributed under the terms of the Creative Common Attribution Non-commercial license (http://creativecommons.org/licenses/by-nc/3.0), which permits use, distribution and reproduction in any medium, provided the original works is properly cited, the use is non-commercial and is otherwise in compliance with the license. 
causes impaired blood flow inside the root, which compromises its function. The symptoms occur in a specific place. Patients experience problems with functioning of the root forming the sciatic nerve, and pinpointing the location of the symptoms can help in evaluating the extent of damage (Faleiros 2009). Pathology of the intervertebral disc is most commonly a cause of spinal pain, though abnormalities may occur also in ligaments, muscles and tendons. The dehydration of the intervertebral disc results in degeneration of the annulus fibrosus. In time, that leads to tearing, first in the external, and later on in the internal layers of the annulus, which allows for the dislocation of the nucleus pulposus, herniation, or even sequestration. The anatomical changes resulting from degenerative disc disease cause symptoms such as pain in the lumbar section of the spine, prevertebral pain and pain radiating to the lower limb peripheries (Fuso et al. 2013).

Treatment is based on many factors, but mainly on the clinical state of the patient and the extend of neurological damage. However, at all times the treatment is preceded by proper medical diagnosis of the disorder (Lichert 2013).

Main symptoms of degenerative disc disease are connected to the occurrence of:

1. pain in the lumbosacral section of the spine (the first and dominating symptom). The onset of the disease is quite acute, and later passes into a chronic form;

2. pain originating in the nerve root - the neuralgia of sciatic nerve - which is experienced along the entire length of the nerve, starting around the buttocks, through the thigh, knee, calf and down the side of the foot all the way to the toes;

3. pain accompanied by feeling impairment, usually superficial and in the form of paraesthesia, but also weakening along the area of distribution of the damaged nerve root;

4. weakening of the tendon reflexes and symptoms of muscular dystrophy, reflected by a reduced circumference of the affected limb;

5. increased tension of prevertebral muscles on the side where nerve roots are compressed;

6. pain between the scapulae or even in the neck, created, so to speak, by the relocation of the inflammatory reaction along the dural sac, from the lumbar section towards the neck. This symptom is rare;

7. central or one-sided pain resulting from the mechanic compression of the dural sac and the nerve root. This symptom occurs usually in younger patients.

A previous damage or long lasting strain may exacerbate the symptoms or quicken the sudden onset of the disease. The course of the disc disease is rather typical, and begins with an abrupt pain resulting from a sudden movement or lifting an object more than $10 \%$ heavier than the patients weight, with an additional rotation of the spine (Wójcik et al. 2013). Degenerative disc disease is often connected with the impairment of the spine's mobility, which results in an increased fatigability of the spine. The disease is often accompanied by different types of pain, often dull, extensive, hard to identify. After a while, however, the pain concentrates and is evidently experienced around the lumbar section of the spine.

Acute and sub-acute phase of the disease is accompanied by pain radiating along the length of the sciatic nerve. The extensiveness of the radiation depends on the level of damage to the nerve root. This may be evaluated during a clinical trial by pressuring Valleix points and judged by the level of movement restriction in the leg caused by pain during the positive Romberg's test (Orłowski 2003). Resting in neutral spine position, with bent hips and knees reduces the pain in the lumbosacral section. In rare cases a severe impairment of motor functions in the legs and sphincter paralysis may occur in the course of the disease. Such patients should undergo immediate surgical treatment (Celik et al. 2012).

\section{Conservative treatment}

Above all, proper treatment of sciatic neuritis requires identifying the source of irritation of the root of the sciatic nerve through imaging examination of the lumbosacral section of the spine, such as CT (computed tomography) or MRI (magnetic resonance imaging) (Khyzhniak et al. 2013).

Conservative treatment of sciatic neuritis is based mainly on pharmaceutical treatment, in which NSAIDs (Nonsteroidal anti-inflammatory drugs) and other analgesics are used. Conservative treatment involves also suitable rehabilitation - manual therapy or neuromobilisation. Some passive methods of treatment, such as applying strain-relieving positions of the spine, are also employed.

Like in the case of many other disorders, methods such as acupuncture, acupressure, massage, gymnastics and relaxation - e.g. yoga, are used widely in the treatment of sciatic neuritis. Despite the lack of unequivocal clinical research asserting their effectiveness, they can be used additionally with some patients because of their apparent harmlessness.

Acupressure and acupuncture - those two techniques of massage are very similar, and they originated in 
Chinese medicine. The basis for those methods is vital energy qi, as well as yin and yang. They help not only to relieve muscle tension, but also to treat some disorders. Acupuncture consists in piercing specific points on the body with very thin needles. Acupressure consists in pressuring those points. In case of degenerative disc disease, both those methods have a purely analgesic effect (Yeh et al. 2009).

Through a set of stretching and posture-improving exercises, yoga can improve the elasticity and mobility of postural muscles and some other muscles, e.g. the piriformis muscle, which is a common cause of sciatic neuritis. Relaxation, which is a part of yoga, may also help to reduce the perceived pain. Yoga may help retain motor functions, reduce the intensity of pain, and treat depression, which is very important in treating chronic diseases (Jeng et al. 2011).

General cryotherapy has become popular in recent years. It consists in exposing the entire body of the patient to very low temperatures $\left(-160\right.$ to $\left.-100^{\circ} \mathrm{C}\right)$ for a short period of time (maximum 3 minutes). Contrary to local cryotherapy, properly executed general cryotherapy does not damage tissue. This method is also called cryostimulation, because its aim is to put the organism under physiological stress (Gusarova et al. 2000).

Using ultrasounds on the paraspinal space brings reasonably good results. It causes the soft tissue to relax, and increases the blood flow in the treated region thanks to deep heat penetration. This improves the tissue nutrition and helps flush out metabolic waste (Licciardone 2013).

Magnet therapy has been used for some time in treating diseases of the lower section of the spine. It consists in exposing live tissue to an alternating magnetic field, which causes changes in the ionic balance. The result of that is a very positive change in metabolism, which is used widely in treating medical conditions such as chronic low back pain (Khoromi et al. 2007).

Transcutaneous Electrical Nerve Stimulation (TENS) is a method used in conservative treatment of degenerative disc disease. Electrical Stimulation is a clinically attested, non-invasive, non-pharmaceutical and effective method of treating pain. It is widely used to alleviate both chronic and sharp pain. TENS consists in using electrodes to send electrical impulses of low amplitude through skin to peripheral nerves, which are responsible for perceiving temperatures. Through those nerves the signal is carried on to the synapses of the spinal cord, where it serves two purposes:

1. As a signal that blocks a nerve impulse, which conveys information to the brain about pain so that the brain does not receive any data of location or the intensity of pain. This effect is caused by high-frequency TENS impulses. Blocking of pain impulses does not damage a nerve structure but it "deceives" the pain information mechanism. The blocking mechanism of a nerve impulse, conveying a signal about pain, was scientifically approved in the 1960s as the gate control theory of Melzack and Wall. Group C nerve fibres convey a signal from an electrostimulator faster than group A nerve fibres so that the signal from a stimulator wins in the contest for an access to the hypothalamus.

2. As an initiator of formulation of natural analgesics, beta endorphins, which have an analgesic effect similarly strong as morphine (or other opioid compounds). This mechanism is caused by low-frequency or bursts impulses.

TENS does not heal causes of pain but it significantly relieves them and an analgesic effect lasts longer (Liebano 2013).

Chiropractic is, on the other hand, a form of a manual therapy, which can be also helpful in treatment of sciatica. To put it in simple terms, it uses certain manoeuvers that reflect the anatomical shape of the spine.

\section{Surgical treatment}

In a case of a hernia or an inner canal protrusion, in the majority of patients with lumbar discopathy one of the most effective methods of surgical treatment is a typical fenestration. Such an operation enables not only a hernia or a sequestrum removal but also a revision of intervertebral space. A wide surgical access enables a full decomposition of a nerve root on his relatively long course, an excision of sometimes present steophytes and also allows the execution of foraminotomy associated with a stenosis of the foramen (Silva et al. 2013).

In case of degenerative disc disease, hemilaminectomy is performed less frequently. It also allows decompression of the nerve tissue but it is associated with damage to bone tissue and thereby extends time of patient's full recovery (Liu et al. 2013).

Currently available methods of surgical treatment:

- standard removal of the intervertebral disc - all intervertebral disc removal techniques give comparable results. It seems to be of importance to appropriate classify noslogical image to the right kind of neurosurgery;

- microsurgical removal of the intervertebral disc involves the use of an optical microscope that significantly improves accuracy and precision of the procedure. The method is less useful in the presence of additional pathology that requires a wider field of a surgical intervention; 
- percutaneous techniques impacting on the structure of the intervertebral disc - different methods based on the US laser technology, high-frequency waves mainly used for the type of relentless spine pain syndrome of a fibrous ring of the intervertebral disc. Methods do not apply in the case of herniation of the spinal canal stenosis developed in discopathy and in patients with a visible sequestration of fragments of the nucleus pulposus in diagnostic imaging;

- dissolution of the intervertebral disc by injection of an enzyme - a method virtually abandoned due to new technologies, but mainly because of dangerous complications related to the destructive action of chymopapain not only on the structure intended for removal;

- numerous methods with use of ceramic, polymer, carbon, fixed or mobile implants are a subject of plentiful clinical observations. After many years of observation, however, it cannot be said they are better than previously used techniques and they are definitely far more expensive for the health care system.

\section{Indications for surgical treatment}

Absolute indication for surgical removal of herniated nucleus pulposus, even on-call duty, is its sudden onset, massive pain syndrome undergoing the symptoms of paralytic seizure and confirmed the light of the spinal canal for diagnostic imaging. (Khyzhniak et al. 2013). The indications for a planned surgery is complete diagnostic imaging and ineffective conservative treatment or progressive symptoms of pain and neurological symptoms. Conservative treatment in such cases should last at most a few weeks or months (Iversen et al. 2013).

Relative indications for surgical removal of the intervertebral disc concern patients with evident symptoms of pain, occurring, however, periodically and relatively seldom, e.g. 2 - 3 times a year. A similar dilemma arises in case of established, yet limited sensory neurological deficits, less frequently in motor deficits. A significant problem occurs, however, in patients with natural conversion of hernia nucleus pulposus into degenerative changes with a reoccurrence of stenosis, which further involves a long duration that significantly worsens prognosis even in the event of surgical treatment (Willems 2013).

\section{Aim of the study}

The aim of this study is to review the current literature on the effects of treatment of patients diagnosed with discectomy in the lumbo-sacral spine.

\section{Material and methods}

A method of descriptive analysis was used. The study was conducted on the basis of an analysis of literature from the MEDLINE and EMBASE database available in the last ten years. Cases of conservative treatment in patients with sciatica caused by discectomy and a similar group of patients undergoing surgery were analysed.

\section{Discussion}

The intervertebral disc degeneration allows for a dislocation of the nucleus pulpous with formation of a hernia but it also leads to secondary lesions within the same vertebrae and intervertebral joints. In course of a longstanding pathological process it comes to an irreversible formation of osteophytes, which cause spinal stenosis and intervertebral foramina, thus to so-called secondary stenosis.

In the case of both conservative and surgical treatment the goal is to reduce or to remove pain and increase functionality of the spine, which directly translates into an increase in overall physical fitness. Both criteria were taken into account when analysing the material.

Traditional "open" surgery of the spine has been used since the 1930s. In Poland, it is one of the most popular methods of surgical treatment. However, it is much more invasive and after such a surgery a patient returns much longer to full physical fitness.

Surgical treatment of disc disease in the lumbar spine is in the present times associated with microdiscectomy. (Kimbal 2013). Discectomy with implantation of a spinal stabilization system is reserved for the most advanced forms of slipped disc. Minimally invasive percutaneous treatment of intervertebral disc degeneration raises many doubts among surgeons treating degenerative disc disease. An important advantage of percutaneous methods is shortened time of hospitalization and rehabilitation. By reducing scope of interference in paraspinal tissue and spinal canal structures the possibility of deepening instability of the spine is minimized and the risk of postoperative scar formation is reduced. Minimally invasive percutaneous treatment of discopathy is 
becoming more and more popular among both patients and surgeons. As in any method of treatment, the clinical effect is dependent on correct classification. Percutaneous methods are used for treatment of less advanced forms discopathy. As a result of increasing prevalence of diagnostic imaging, discopathy is detected in the early stages of development. In order to qualify for a surgery procedure, conservative methods of treatment must be exhausted. Many authors consider 6-month ineffective rehabilitation for a basic eligibility criterion. Minimally invasive percutaneous treatment of discopathy should not be considered as an alternative to the conventional spinal surgery. They often come between the classical conservative treatment and surgery of the spine. Due to various clinical indications for percutaneous and classical surgery, often debated demand to conduct prospective studies, comparing effects of treatment, seems to be more and more difficult to achieve (Manchikanti 2013).

Most of authors reported that after the conservative treatment of patients with lumbar discectomy with sciatica, $20 \%$ of patients needed to again contact a doctor about a relapse within 12 months of health improvement. The analysis showed that aggravation of symptoms before treatment is an important factor affecting the recovery rate. According to current studies, satisfactory short-term results of conservative treatment in patients with symptoms of a lumbar herniated disc support the possibility of their return to physical activity. Results of the conservative treatment were not dependent on the intensity of pain. Subjective symptoms before treatment seem to be a key factor in qualification for conservative treatment (Iwamoto et al. 2011).

The Dutch randomized study was conducted using the Roland Morris Disability Questionnaire, the visual analogue scale (VAS) for the lower limbs and the back and the individual Likert scale helpful in the evaluation of full recovery. Despite at least 6 months conservative treatment, $46 \%$ of patients were qualified for surgery due to intensification of pain in lower limbs and disability. Prolonged conservative treatment may be an opportunity for patients to reduce pain and disability but it also constitutes risk of delayed surgery after long suffering associated with sciatica. An age of 40 and strong back pain radiating into lower limbs support implementation of surgical treatment (Lequin et al. 2013).

Prognostic factors that qualify to surgical treatment of patients with sciatica include obesity and advanced age. (Jensen et al. 2013).

There are discrepancies in the literature as to whether pain increases or decreases in the elderly and whether it is related to gender. There is no definite proof that the prevalence of pain radiating to lower limbs is associated with age. Complementary therapies have proved to be of certain efficiency among the elderly, including acupuncture, transcutaneous electrical nerve stimulation (TENS) and massage (Abdulla et al. 2013).

The comparison of a combination of physiotherapy and traction with physiotherapy used alone or traction compared with other treatments resulted in very weak evidence that traction can affect the intensity of pain, physical fitness of a patient or the improvement of peripheral nerves conduction. These results indicate that traction, either alone or in combination with other treatments, have little or no effect on the intensity of pain, functional status of a patient and general improvement of peripheral nerve conduction. The effects observed in this study are small and not clinically relevant. (Wegner et al. 2013).

On the basis of a randomized clinical trial conducted on 141 patients suffering from neuropathic pain diagnosed with sciatica, effects of adjuvant drug therapy on the conservative treatment were analysed. In case of neuropathic pain, pharmacological treatment is the treatment by choice, traditional conservative treatment is, however, a complementary method which allows to achieve better therapeutic efficacy (Di Piero, Settembre 2013).

The method commonly used in treatment of pain syndromes in the lower spine is a massage. The use of massage seems to have a positive impact on a reduction in disability, the effects of its use, however, are shortterm but for majority of patients anti-depressant (Keller 2012).

Other studies have argued in favour of effectiveness of magnetic field in treatment of lumbar disc herniation. Forty patients suffering from lumbar radiculopathy were randomly assigned to one of two groups. The study group included 20 patients treated with a magnetic field therapy and the control group consisted of 20 patients treated with placebo. Both groups were assessed before treatment and three weeks after it with a use of the Visual Analogue Scale VAS, somatosensory evoked potentials for selected dermatomes and a modified Oswestry Disability Questionnaire (OSW). The group of patients undergoing magnetic field therapy achieved significantly better therapeutic effects. In addition to clinical improvement in nerve roots symptom, the magnetic field also appears to be effective in reduction in nerve root compression (Omar et al. 2012).

Grubisić and colleagues conducted research on the effectiveness of ultrasound therapy in patients with chronic lumbar pain lasting more than three months. Patients were divided into two groups, whereas one was treated only with kinesitherapy and the other with kinesitherapy and sonication treatment. The VAS and the Schober test were used in research. The analysis of the results before and after beginning of the treatment found that ultrasound therapy is effective in reduction in the intensity of pain but does not provide improvement in terms of the functional capability of the lumbar spine in patients with chronic back pain (Grubisic et al. 2006). 
Croatian researchers conducted a retrospective analysis of the effectiveness of surgical and conservative treatment in 100 patients with lumbar radicular syndrome. The Lasègue test and the hybrid Lesegue test were performed in patients; neurological deficit was also evaluated. According to established criteria, all patients had indications for surgical treatment. However, 50 patients refused surgery and as a result were treated conservatively. Data were evaluated at the beginning of the treatment, after 2 months and then after 7 and 18 months. The results of the treatment in all periods supported surgery in majority of specialized research. Better results of the treatment were obtained in the group of patients who underwent surgery. Fast reduction in symptoms is the main advantage of this method. Patients whose pain is treated conservatively and do not have extensive neurological disorders may decide to delay surgery (Hadžić et al. 2013).

Other researchers also addressed the issues of the effectiveness of spinal surgery compared to conservative treatment of lumbar herniated disc. Of the 127 patients with lumbar discectomy, 72 were treated only conservatively, whereas the remaining 55 underwent microdiscectomy. Two years after the completion of treatment as well as before its beginning, the visual analogue scale (VAS) and the Oswestry Disability Index (ODI) together with the Short Form-36 Health Survey (SF-36) were used in both groups for the purpose of the analysis. In both groups, results of the treatment were more favourable compared to baseline values and fluctuated within similar limits. Statistical analysis showed that there were no significant differences between the groups. In conclusion, this study shows that both methods lead to equivalent clinical effects (Kafchitsas et al. 2014)

There were also attempts undertaken to evaluate pain of the lumbar spine and lower limbs by means of the visual analogue scale (VAS) in pre- and postoperative management of patients with sciatica. Intervertebral disc surgery resulted in a significant reduction in pain threshold in each patient. The whole group of patients was examined six months later after a surgery. In the study group, a full clinical improvement was achieved with the VAS within the normal range (Zub et al. 2013).

\section{Conclusions}

A review of the current literature on the effects of treatment of people diagnosed with discectomy in the lumbo-sacral spine shows that:

1. Conservative treatment reduces the intensity of pain but does not improve functional capabilities of the spine.

2. Surgical treatment gives a rapid reduction in symptoms but long-term effects of such a treatment do not appear to be superior to conservative treatment, surgery may furthermore be associated with serious complications.

3. Aggravation of symptoms is an adverse predictor for all methods of treatment.

4. The degree of symptoms aggravation before treatment is an important factor affecting the rate of patient's recovery.

\section{References:}

1. Abdulla A., Adams N., Bone M., Elliott A.M., Gaffin J., Jones D., Knaggs R., Martin D., Sampson L., Schofield P. (2013), Guidance on the management of pain in older people. Age Ageing. 42 Suppl 1: 1-57.

2. Celik E.C., Kabatas S., Karatas M. (2012), Atypical presentation of cauda equina syndrome secondary to lumbar disc herniation. J Back Musculoskelet Rehabil. 25(1): 1-3.

3. Dagenais S. (2013), Why not everyone with low back pain chooses chiropractic care. J Can Chiropr Assoc. 57(1): 6-9.

4. Di Pierro F., Settembre R. (2013), Safety and efficacy of an add-on therapy with curcumin phytosome and piperine and/or lipoic acid in subjects with a diagnosis of peripheral neuropathy treated with dexibuprofen. $\mathrm{J}$ Pain Res. 3;6: 497-503.

5. Faleiros A.T., Resende L.A., Zanini M.A., Castro H.A., Gabarra R.C. (2009), L4-L5-S1 human dermatomes: a clinical, electromyographical, imaging and surgical findings. Arq Neuropsiquiatr. 67(2A): 265-267.

6. Fuso F.A., Dias A.L., Letaif O.B., Cristante A.F., Marcon R.M., de Barros T.E. (2013), Epidemiological study of cauda equina syndrome. Acta Ortop Bras. 21(3): 159-162.

7. Grubisić F., Grazio S., Jajić Z., Nemcić T. (2006), Therapeutic ultrasound in chronic low back pain treatment. Reumatizam. 53(1): 18-21.

8. Gusarova S.A., Kuznetsov O.F., Gorbunov F.E., Maslovskaia S.G. (2000), The methodological aspects of using cryomassage on patients operated on for discogenic neuropathies. Vopr Kurortol Fizioter Lech Fiz Kult. (4): 20-2.

9. Hadžić E., Dizdarević K., Hajdarpašić E., Džurlić A., Ahmetspahić A. (2013), Low back and lumbar radicular syndrome: comparative study of the operative and non-operative treatment. Med Glas (Zenica). 10(2): 309-315. 
10. Iversen T., Solberg T.K., Romner B., Wilsgaard T., Nygaard Ø., Waterloo K., Brox J.I., Ingebrigtsen T. (2013), Accuracy of physical examination for chronic lumbar radiculopathy. BMC Musculoskelet Disord. 9;14: 206.

11. Iwamoto J., Sato Y., Takeda T., Matsumoto H. (2011), Return to play after conservative treatment in athletes with symptomatic lumbar disc herniation: a practice-based observational study. Open Access J Sports Med. 2: 25-31.

12. Jeng C.M., Cheng T.C., Kung C.H., Hsu H.C. (2011), Yoga and disc degenerative disease in cervical and lumbar spine: an MR imaging-based case control study. Eur Spine J. 20(3): 408-13.

13. Jensen O.K., Stengaard-Pedersen K., Jensen C., Nielsen C.V. (2013), Prediction model for unsuccessful return to work after hospital-based intervention in low back pain patients. BMC Musculoskelet Disord. 14: 140-146.

14. Keller G. (2012), The effects of massage therapy after decompression and fusion surgery of the lumbar spine: a case study. Int J Ther Massage Bodywork. 5(4): 3-8.

15. Khoromi S., Blackman M.R., Kingman A., Patsalides A., Matheny L.A., Adams S., Pilla A.A., Max M.B. (2007), Low intensity permanent magnets in the treatment of chronic lumbar radicular pain. J Pain Symptom Manage. 34(4): 434-45.

16. Khyzhniak M.V., Chuvashova O.Iu., Novakovych K.S. (2013), Instrumental diagnosis of recurrent lumbar spine herniated discs in different observation periods. Klin Khir. (8): 69-71.

17. Kimball J., Yew A., Lu D.C. (2013), Minimally invasive surgery for lumbar microdiscectomy. Neurosurg Focus. 35(2 Suppl): 7-9.

18. Koes B.W., van Tulder M.W. (2007), Diagnosis and treatment of sciatica. BMJ. 334(7607): 1313-1317.

19. Lequin M.B., Verbaan D., Jacobs W.C., Brand R., Bouma G.J., Vandertop W.P., Peul W.C. (2013), Leiden-The Hague Spine Intervention Prognostic Study Group; WCP; BWK; RTWMT; WBvdH; RB. Surgery versus prolonged conservative treatment for sciatica: 5-year results of a randomised controlled trial. BMJ Open. 3(5): 119-127.

20. Licciardone J.C. (2013), Osteopathic manual treatment and ultrasound therapy for chronic low back pain: an illustration of osteopathic semantic confusion. Author reply. J Am Osteopath Assoc. 113(9): 661-2.

21. Lichert F. (2013), Classification of traumatic intervertebral disc lesions. Rofo. 185(11): 1026.

22. Liu K., Shi J., Jia L., Yuan W. (2013), Surgical technique: Hemilaminectomy and unilateral lateral mass fixation for cervical ossification of the posterior longitudinal ligament. Clin Orthop Relat Res. 471(7): 2219-2224.

23. Manchikanti L., Singh V., Falco F.J., Calodney A.K., Onyewu O., Helm S. 2nd, Benyamin R.M., Hirsch J.A. (2013), An updated review of automated percutaneous mechanical lumbar discectomy for the contained herniated lumbar disc. Pain Physician. 16(2 Suppl): 151-184.

24. Melancia J.L., Francisco A.F., Antunes J.L. (2014), Spinal stenosis. Handb Clin Neurol. 119: 541-9.

25. Mixter W.J., Barr J.S. (1934), Rupture of the intervertebral disc with involvement of the spinal canal. $\mathrm{N}$ Engl J Med 211: 210-215.

26. Omar A.S., Awadalla M.A., El-Latif M.A. (2012), Evaluation of pulsed electromagnetic field therapy in the management of patients with discogenic lumbar radiculopathy. Int J Rheum Dis. 15(5): 101-8.

27. Orłowski W. (2003), Zarys ogólnej diagnostyki lekarskiej. PZWL, Warszawa.

28. Rüttimann B. (1990), A historical approach to herniated disc. Schweiz Rundsch Med Prax. 79(25): 791-796.

29. Silva P.S., Pereira P., Monteiro P., Silva P.A., Vaz R. (2013), Learning curve and complications of minimally invasive transforaminal lumbar interbody fusion. Neurosurg Focus. 35(2): 7.

30. Stienen M.N., Surbeck W., Tröhler U., Hildebrandt G. (2013), Little-known Swiss contributions to the description, diagnosis, and surgery of lumbar disc disease before the Mixter and Barr era. J Neurosurg Spine. 84: 1138-1145.

31. Wegner I., Widyahening I.S., van Tulder M.W., Blomberg S.E., de Vet H.C., Brønfort G., Bouter L.M., van der Heijden G.J. (2013), Traction for low-back pain with or without sciatica. Cochrane Database Syst Rev. 8: 301-308.

32. Willems P. (2013), Decision making in surgical treatment of chronic low back pain: the performance of prognostic tests to select patients for lumbar spinal fusion. Acta Orthop Suppl. 84(349): 1-35.

33. Wójcik G., Bulikowski W., Piskorz J., Stawińska T., Sokołowska B. (2013), Computed tomography and stretch tests in diagnosing sciatica as a method for ensuring patient well-being, In: W. Kurlej (ed.), Health and wellness, Lublin, NeuroCentrum, 223-235.

34. Yeh C.C., Wu C.T., Huh B.K., Lee S.M., Wong C.S. (2009), Collateral meridian acupressure therapy effectively relieves postregional anesthesia/analgesia backache. South Med J. 102(11): 1179-82.

35. Zhang Y., Sun Z., Liu J., Guo X. (2008), Advances in susceptibility genetics of intervertebral degenerative disc disease. Int J Biol Sci. 2;4(5): 283-290.

36. Zub L.W., Szymczyk M., Pokryszko-Dragan A., Bilińska M. (2013), Evaluation of pain in patients with lumbar disc surgery using VAS scale and quantitative sensory testing. Adv Clin Exp Med. 22(3): 411-419.

Submitted: 18.03 .2014

Accepted: 19.03.2015 